\title{
An explicit linearized state-space technique for accelerated simulation of electromagnetic vibration energy harvesters
}

\author{
Tom J. Kazmierski, Senior Member, IEEE, Leran Wang, Member, IEEE, Bashir M. Al-Hashimi, Fellow, IEEE, \\ and Geoff V. Merrett, Member, IEEE
}

\begin{abstract}
Vibration energy harvesting systems pose significant modeling and design challenges due to their mixed-technology nature, extremely low levels of available energy and disparate time scales between different parts of a complete harvester. An energy harvester is a complex system of tightly coupled components modeled in the mechanical, magnetic as well as electrical analog and digital domains. Currently available design tools are inadequate for simulating such systems due to prohibitive CPU times. This paper proposes a new technique to accelerate simulations of complete vibration energy harvesters by approximately two orders of magnitude. The proposed technique is to linearize the state equations of the system's analog components to obtain a fast estimate of the maximum step-size to guarantee the numerical stability of explicit integration based on the Adams-Bashforth formula. We show that the energy harvester's analog electronics can be efficiently and reliably simulated in this way with CPU times two orders of magnitude lower than those obtained from two state-of-the art tools, VHDL-AMS and SystemC-A. As a case study, a practical, complex microgenerator with magnetic tuning and two types of power processing circuits have been simulated using the proposed technique and verified experimentally.
\end{abstract}

Index Terms-Energy harvesting, state-space technique, simulation acceleration, tunable microgenerator, DC-to-DC converter.

\section{INTRODUCTION AND MOTIVATION}

Recently, significant research interest has been attracted to the development of energy harvesters. The aim is to address the energy conservation issue in the fast growing area of mobile electronics and several emerging applications including wireless sensor networks [1-4]. Energy harvesters capture small amounts of energy from the environment, e.g. solar [5], thermal [6], RF [7] or kinetic energy [8], and use it to power miniature wireless autonomous devices. Vibration-based kinetic energy harvester are used in many commercial applications since mechanical vibrations are widely present [9]. There are three main transduction mechanisms in vibrationbased energy harvesting: electromagnetic, piezoelectric and electrostatic, each of which has various implementations. An electromagnetic microgenerator normally generates low voltage and high current output while piezoelectric and electrostatic ones normally generate high voltage and low current output [9]. Typically, practical implementations of the above transduction mechanisms produce output voltages unsuitable to directly power electronic circuitry. The amplitude of the AC voltage generated by a miniaturized electromagnetic generator

Copyright (C)2011 IEEE. Personal use of this material is permitted. However, permission to use this material for any other purposes must be obtained from the IEEE by sending an email to pubs-permissions@ieee.org. is typically too small [8]. On the other hand, output voltages from electrostatic and piezoelectric devices are normally too high (over $100 \mathrm{~V}$ in some cases) [10]. For this reason external analog circuits are often employed to regulate (i.e boost or step down) the output voltage [11]. A storage element, such as a supercapacitor or a rechargeable battery, is used in such energy harvesting systems to store the generated energy. The load circuitry will only wake up and perform computations when enough energy has been accumulated [12]. Most of the reported electromagnetic microgenerator designs are based on a spring-mass-damper system with a characteristic resonant frequency. These devices normally have a high Q-factor and generate maximum power when their resonant frequency matches the dominant frequency of the input ambient vibration [13]. Consequently, the output power generated by the microgenerator drops dramatically when there is a difference between the dominant ambient frequency and the microgenerator's resonant frequency. Tunable microgenerators, which can adjust their own resonant frequency through mechanical or electrical methods to match the input frequency, have become an emerging area and are attracting great research interest [14]. The key components of a tunable energy harvester system are shown in Fig. 1).

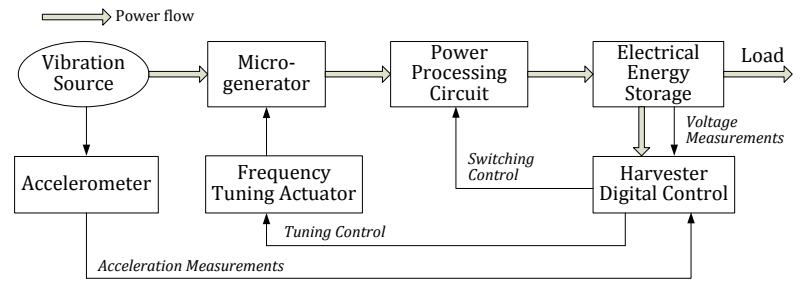

Fig. 1. Components of a tunable energy harvesting system [15].

Although research into new energy harvesting devices is fast developing, there has been little reported work on modeling and simulation methodologies for such mixed-technology systems. Most of the existing energy harvester models describe the mechanical, magnetic and and electrical parts separately and separate software tools are used to produce optimal designs of the individual parts $[16,17]$. But as recently demonstrated [18], the combination of optimal component blocks may not lead to an optimal integrated system. In Fig. 1, the microgenerator and the tuning actuator are mainly mechanical components and the rest of the system consists of analog and digital electrical circuits. The most common approach seen in literature is to use Finite Element Modeling (FEM) tools to simulate the mechanical part [19-21]. Zhu et al. have de- 
veloped an electromagnetic microgenerator which is tuned by magnetic force [19] and used ANSYS (http://www.ansys.com) to determine the resonant frequency of the microgenerator cantilever beam and to simulate the magnetic field between the two tuning magnets. Hohlfeld et al. have presented the design of an electrostatic microgenerator which is tuned by electrostatic force [20]. The simulation of the electrostatic field was carried out by CoventorWare (http://www.coventor.com). However, FEM tools cannot be used directly to simulate and optimize complete energy harvesting systems because they cannot incorporate the system's electrical components. As for the electrical part, SPICE simulators are widely used for circuit simulation [22] and Cadence/SYNOPSYS tools are popular in IC design [23]. A hardware description language (HDL) can model both the mechanical and electrical parts of an energy harvester accurately [18]. The limitation of the HDL simulation based approach is that available HDL tools are inadequate for simulating complete, tunable energy harvester systems due to prohibitive CPU times. A single simulation of a supercapacitor charging may take more than 10 hours to finish [15]. There are two reasons for the long simulation times. Firstly with reference to Fig. 1, vibration energy harvesters typically have an input frequency of tens or hundreds of hertz in line with the typical environment vibrations encountered, requiring a fine simulation time-step of less than a millisecond. If a switching mode DC-DC converter is employed as the power processing circuit, the simulation time-step needs to be further reduced due to the fast switching behavior [24]. In contrast, large supercapacitors used in energy harvesting systems require many hours to fully charge, given the low levels of power generated by typical vibration microgenerators. Secondly, all of the existing HDL simulators use implicit differentiation techniques based on Newton-Raphson iterations to solve the underlying differential-algebraic equations (DAEs) that model the energy harvester's analog part. Implicit differentiation and Newton-Raphson iterations are reliable and numerically stable [25] but, when applied to a complex mixedtechnology energy harvester system, they lead to long CPU times. Therefore new techniques to accelerate the simulation of energy harvesters are needed, which is the main purpose of the research presented in this paper. We take advantage of the inherent passivity of the energy harvester analog electronics and propose to use a linearized state-space equation formulation. The proposed technique is fast and the linearization enables efficient control of numerical stability in explicit integration. Simulations of the complete energy harvester to calculate the power transfer efficiency have been carried out in several scenarios. Results in Section IV show a good match with experimental measurements and the proposed technique has been found to reduce simulation times by about two orders of magnitude (from 8 hours to less than 4 minutes in one of tuning scenarios) with no loss of accuracy.

\section{Proposed linearized state-Space technique}

Consider the following state equation of a non-linear, passive dynamic system:

$$
\dot{\boldsymbol{x}}(t)=\boldsymbol{f}(\boldsymbol{x}(t), t) ; \quad \boldsymbol{x}(0)=\boldsymbol{x}_{0}
$$

where $\boldsymbol{x} \in \mathbb{R}^{N}$ is the vector of $N$ state variables, $t \in[0, \infty)$, $\boldsymbol{x}_{0}$ is the initial condition and $\boldsymbol{f}: \mathbb{R}^{N} \times \mathbb{R}$.

The linearized system at time point $t_{k}, k=0,1, \ldots$ is:

$$
\dot{\boldsymbol{x}}(t)=\boldsymbol{J}_{k}\left(\boldsymbol{x}(t)-\boldsymbol{x}_{k}\right)+\boldsymbol{f}\left(\boldsymbol{x}_{k}, t\right)+\mathcal{O}\left(\left(t-t_{k}\right)^{2}\right)
$$

where $\boldsymbol{x}_{k} \equiv \boldsymbol{x}\left(t_{k}\right)$ and $\boldsymbol{J}_{k}$ is the Jacobian of $\boldsymbol{f}$ at $t_{k}$. Since the system is passive, the eigenvalues of $\boldsymbol{J}_{k}$ have negative real parts [25]. The local linearization error $\left(L L E_{k}\right)$ introduced at time point $t_{k}$ is:

$$
L L E_{k}=\left\|\boldsymbol{x}^{*}\left(t_{k}\right)-\boldsymbol{x}_{k}\right\|
$$

where $\boldsymbol{x}^{*}\left(t_{k}\right)$ are the accurate values and $\boldsymbol{x}\left(t_{k}\right)$ are the approximate values of $\boldsymbol{x}(t)$ obtained due to linearization at $t_{k}$. The $L L E$ is caused by the rejection of the Taylor expansion terms of order higher than the first. Hence, the $L L E$ is proportional to $\left(t-t_{k}\right)^{2}$ as illustrated in equation (2).

\section{A. Fast step-size estimate for stability control}

The linearized state equation (2) can be solved in a fast, explicit march-in-time integration process without NewtonRaphson iterations. State-of-the-art circuit simulators use implicit, rather than explicit integration, to assure numerical stability. The implicit approach has proved reliable in circuit analysis where differential equations are commonly 'stiff' [25], i.e. contain multiple time scales, with both rapid transients and slow dynamics. As systems exhibiting stiff behavior can have eigenvalues with large negative real parts as well as eigenvalues with small negative real parts, in an explicit integration process the step-size must be limited not just to control accuracy of the solution but primarily to ensure stability [25]. Stability control is difficult as it requires estimates of the maximum eigenvalue $\lambda_{k}$ of the Jacobian $\boldsymbol{J}_{k}$ at each step size which is typically a time consuming process [25]. In our proposed technique for energy harvester simulations we take advantage of the system's passivity and use a fast method of estimating the maximum allowed step size directly from the Jacobian entries.

Theorem: (Fast stability control for diagonally dominant Jacobians) The Adams-Bashforth integration formula of order $p$ applied to the following set of ODEs:

$$
\dot{\boldsymbol{x}}(t)=\boldsymbol{A} \boldsymbol{x}(t)
$$

where $\boldsymbol{A}$ is negative definite and diagonally dominant, is numerically stable if the integration step size $h$ :

$$
h \leq \frac{1}{\max _{r=1, \ldots, N}\left(\beta_{\max }\left|a_{r, r}\right|\right)}
$$

where $a_{r, r}$ represents the diagonal element in row $r$ of $\boldsymbol{A}$ and $\beta_{\max }=\max \left(\left|\beta_{0}\right|, \ldots,\left|\beta_{p}\right|\right)$ is the modulus of the maximum coefficient of the $p$ - th order Adams-Bashforth formula.

Proof: The Adams-Bashforth integration scheme for equation (4) is:

$$
\boldsymbol{x}_{k+1}=\left(\boldsymbol{I}+h \beta_{0} \boldsymbol{A}\right) \boldsymbol{x}_{k}+h \boldsymbol{A} \sum_{i=1}^{p} \beta_{i} \boldsymbol{x}_{k-i} ; k=1, \ldots,
$$

where $\beta_{i}, \quad i=0, \ldots p$ are Adams-Bashforth coefficients [26]. Errors are damped if $\left\|\boldsymbol{I}+h \beta_{0} \boldsymbol{A}\right\| \leq 1$ and 
||$h \beta_{i} \boldsymbol{A} \| \leq 1, i=1, \ldots, p$. As the Gerschgorin disks of a negative definite and diagonally dominant matrix lie in the lefthand complex plane [27], stability of the integration scheme in equation (6) is achieved when $\left|1-h \beta_{\max }\right| a_{r, r} \mid \leq 1 ; r=$ $1, \ldots, N$, i.e. when the step-size is limited by the condition in equation (5).

The proposed technique is conservative in the sense that step sizes obtained from equation (5) are expected to be smaller than the maximum allowed step sizes calculated from the exact values of the Jacobian's eigenvalues. However, the advantage of our technique is speed as expensive eigenvalue calculations are avoided. Many passive electrical circuits, such as the power conditioning system and the supercapacitor in the case study presented below, have negative definite and diagonally dominant Jacobians. The mechanical part of the microgenerator is a classical mass-spring-damper system:

$$
\frac{d^{2} z(t)}{d t^{2}}+2 \zeta \omega_{0} \frac{d z(t)}{d t}+\omega_{0}^{2} z(t)=0
$$

where $\zeta$ is the damping factor and $\omega_{0}$ - the resonant frequency. In a high-Q vibrating system $\zeta \ll 1$ and the corresponding rows in the Jacobian matrix are not diagonally dominant. However, estimation of the maximum allowed step size to guarantee stability in this case is simple. The state equations of a homogeneous mass-spring-damper system equation (7) can be presented as a set of two first order ODEs:

$$
\left[\begin{array}{l}
\frac{d y(t)}{d t} \\
\frac{d z(t)}{d t}
\end{array}\right]=\left[\begin{array}{cc}
-2 \zeta \omega_{0} & -\omega_{0}^{2} \\
1 & 0
\end{array}\right]\left[\begin{array}{l}
y(t) \\
z(t)
\end{array}\right]
$$

Stability of Adams-Bashforth integration with step size $h$ can be determined from the eigenvalues of the matrix in equation (8) which are $\lambda_{1,2}=-\zeta \omega_{0} \pm 2 \omega_{0} \sqrt{\zeta^{2}-1}$. Hence Adams-Bashforth integration is stable provided the points $h \lambda_{1,2}$ lie within the stability region of the Adams-Bashforth formula used [28]. Specifically, if $\zeta \ll 1$, then $\left|\lambda_{1,2}\right| \approx\left|2 \omega_{0}\right|$ and if the 3rd order Adams-Bashforth formula is used, $h \lambda_{1,2}$ lie within the 3 rd order formula stability region as long as [28]:

$$
2 h \omega_{0}<0.723
$$

This is normally always the case provided the numerical solution covers each vibration period with at least four time points. In the case study of the tunable energy harvester discussed in Section III, the linearized state space formulation proposed above was applied to the harvester's mechanical and electrical analog parts:

$$
\left[\begin{array}{c}
\dot{\boldsymbol{x}}(t) \\
\mathbf{0}
\end{array}\right]=\left[\begin{array}{l}
\boldsymbol{f}_{x}(\boldsymbol{x}(t), \boldsymbol{y}(t)) \\
\boldsymbol{f}_{y}(\boldsymbol{x}(t), \boldsymbol{y}(t))
\end{array}\right]+\left[\begin{array}{c}
\boldsymbol{e}_{x}(t) \\
\mathbf{0}
\end{array}\right]
$$

where $\boldsymbol{x}: \mathbb{R} \longrightarrow \mathbb{R}^{N}$ is the vector of $N$ state variable waveforms, $\boldsymbol{y}: \mathbb{R} \longrightarrow \mathbb{R}^{M}$ are $M$ non-state variable waveforms, the non-linear functions $\boldsymbol{f}_{x}: \mathbb{R}^{N} \times \mathbb{R}^{M} \longrightarrow \mathbb{R}^{N}$ and $f_{y}: \mathbb{R}^{N} \times \mathbb{R}^{M} \longrightarrow \mathbb{R}^{M}$ represent the relationships between the variables of the model and $\boldsymbol{e}_{x}: \mathbb{R} \longrightarrow \mathbb{R}^{N}$ is the vector of excitations. The state variables $\boldsymbol{x}(t)$ are mixedphysical-domain quantities related with energy storage in the model. Examples of state variables are the displacement of the vibrating magnet, its velocity, magnetic flux as well as electrical voltages and currents of the capacitive and inductive components correspondingly. The non-state variables typically represent terminal voltages and currents which connect individual modules of the system as illustrated in Fig. 2.

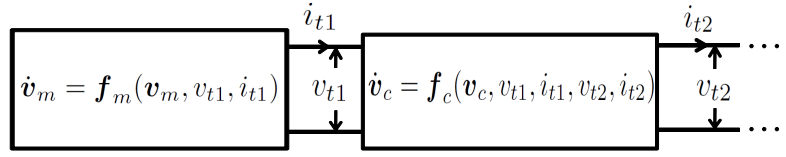

Fig. 2. Energy harvester analog blocks showing state equations and terminal variables.

\section{B. Energy-based stability control}

The passivity property of equation (2) can be used to verify aposteriori, i.e. on completion of each step, whether stability is maintained. Namely, the passivity implies that the homogeneous part of equation (2), i.e. with the external excitations removed:

$$
\dot{\boldsymbol{x}}^{\prime}(t)=\boldsymbol{J}_{k} \boldsymbol{x}^{\prime}(t)
$$

must dissipate energy, i.e. the second norm of the solution of equation (11) must decay in time: $\left\|\boldsymbol{x}^{\prime}\left(t_{k+1}\right)\right\|_{2}<\left\|\boldsymbol{x}^{\prime}\left(t_{k}\right)\right\|_{2}$ for all $t_{k}=0,1, \ldots$. The energies accumulated in the individual components of a kinetic energy harvester comprise: electrical energy stored in capacitors $\frac{1}{2} C v^{2}(t)$, magnetic energy stored in inductors $\frac{1}{2} L i^{2}(t)$, mechanical kinetic energy of the microgenerator's moving mass $m: \frac{1}{2} m \frac{d z(t)}{d t}^{2}$ and the mechanical potential energy of the cantilever beam $\frac{1}{2} k_{s} z^{2}(t)$, where $k_{s}$ is the cantilever spring effective stiffness and $z(t)$ is the relative displacement between the vibrating mass and the base. As the state vector consists of the physical quantities associated with the corresponding energy storage elements, i.e. the capacitor voltages, inductor currents, moving mass velocity and displacement, the second norm of the homogeneous solution $\left\|\boldsymbol{x}^{\prime}\left(t_{k+1}\right)\right\|_{2}=\sqrt{\left.{x^{\prime}}^{2}\left(t_{k+1}\right)+\ldots+{x^{\prime}}_{N}^{2}\left(t_{k+1}\right)\right)}$ at each step is proportional to the total energy stored in the system.

\section{Elimination of terminal variables}

The linearized state-space technique outlined above has an additional advantage of enabling automatic elimination of nonstate variables. The linearized equation (10) at each time-point $t_{k}, k=0,1, \ldots$ is:

$$
\left[\begin{array}{c}
\dot{\boldsymbol{x}}_{\left(t_{k}\right)} \\
\mathbf{0}
\end{array}\right]=\left[\begin{array}{ll}
\boldsymbol{J}_{x x, k} & \boldsymbol{J}_{x y, k} \\
\boldsymbol{J}_{y x, k} & \boldsymbol{J}_{y y, k}
\end{array}\right]\left[\begin{array}{l}
\boldsymbol{x}\left(t_{k}\right) \\
\boldsymbol{y}\left(t_{k}\right)
\end{array}\right]+\left[\begin{array}{c}
\boldsymbol{e}_{x}\left(t_{k}\right) \\
\mathbf{0}
\end{array}\right]
$$

where the Jacobian matrices of the linearized model at the time point $t_{k}$ are: $\boldsymbol{J}_{x x, n}=\frac{\partial \boldsymbol{f}_{x}\left(t_{k}\right)}{\partial x}, \boldsymbol{J}_{x y, k}=\frac{\partial \boldsymbol{f}_{x}\left(t_{k}\right)}{\partial y}, \boldsymbol{J}_{y x, k}=$ $\frac{\partial \boldsymbol{f}_{y}\left(t_{k}\right)}{\partial x}$ and $\boldsymbol{J}_{y y, k}=\frac{\partial \boldsymbol{f}_{y}\left(t_{k}\right)}{\partial y}$.

At each time point $t_{k}$ the non-state variables $\boldsymbol{y}\left(t_{k}\right)$ are eliminated by solving the following linear algebraic equation, i.e the algebraic part of the linearized equation (12):

$$
\boldsymbol{J}_{y y, k} \boldsymbol{y}\left(t_{k}\right)=-\boldsymbol{J}_{y x, k} \boldsymbol{x}\left(t_{k}\right)
$$

Once the variables $\boldsymbol{y}\left(t_{k}\right)$ are calculated at $t_{k}$, the state variables $\boldsymbol{x}(t)$ can be obtained at the next time point $t_{k+1}$ by applying the technique explained above based on an explicit Adams-Bashforth differentiation formula. 


\section{ENERGY HARVESTER COMPONENT MODELS FOR LINEARIZED STATE-SPACE FORMULATION}

In the following subsections, we present the model equations of each component block of the tunable energy harvester (Fig. 1) and show how they can be formulated as linearized state equations. The complete VHDL-AMS and SystemC-A code for the case study models and simulations is available at http://www.holistic.ecs.soton.ac.uk/resources.php.

\section{A. Tunable microgenerator}

Fig. 3(a) shows a diagram of the electromagnetic microgenerator together with its tuning mechanism. The microgenerator is based on a cantilever structure. The coil is fixed to the base, and four magnets (which are located on both sides of the coil) form the proof mass. The tuning mechanism uses magnetic force to change the effective stiffness of the cantilever which leads to a change of resonant frequency. One tuning magnet is attached to the end of the cantilever beam and the other tuning magnet is connected to a linear actuator. The linear actuator moves the magnet to desired position so that the resonant frequency of the microgenerator matches the frequency of the ambient vibration. The control algorithm is modeled as a SystemC digital process described in Section III-D. Fig. 3(b) shows a photo of the microgenerator which is used to validate the proposed technique [29].

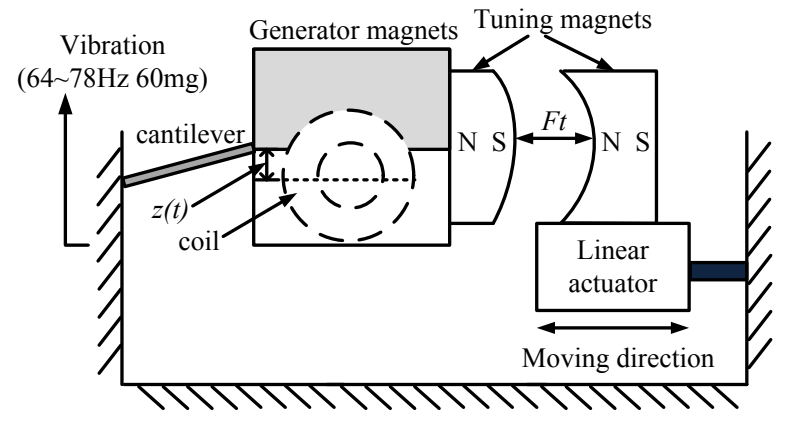

(a) Mechanical part

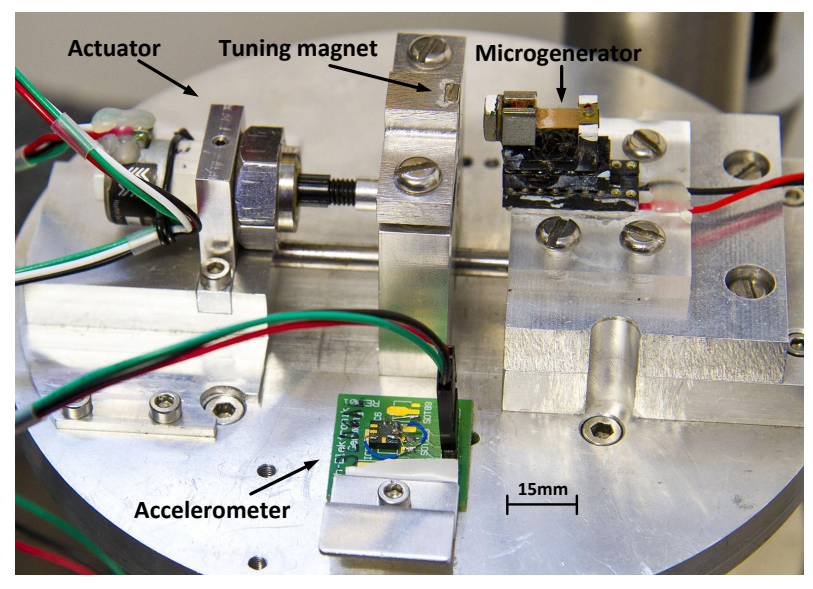

(b) Photo of tunable microgenerator.

Fig. 3. Tunable electromagnetic microgenerator [29].

The dynamic model of the microgenerator is [19]:

$$
m \frac{\mathrm{d}^{2} z(t)}{\mathrm{d} t^{2}}+c_{p} \frac{\mathrm{d} z(t)}{\mathrm{d} t}+k_{s} z(t)+F_{e m}+F_{t_{-} z}=F_{a}
$$

where $m$ is the proof mass, $z(t)$ is the relative displacement between the mass and the base, $c_{p}$ is the parasitic damping factor, $k_{s}$ is the effective spring stiffness, $F_{e m}$ is the electromagnetic force, $F_{t_{-} z}$ is the $z$ component of tuning force $F_{t}$ and $F_{a}$ is the input acceleration force. The resonant frequency $\omega_{0}$ and damping coefficient $\zeta$ are:

$$
\omega_{0}=\sqrt{\frac{k_{s}}{m}} \quad \text { and } \quad \zeta=\frac{c_{p}}{2 \sqrt{m k_{s}}}
$$

The electromagnetic voltage generated in the coil is:

$$
V_{e m}=-\Phi \frac{\mathrm{d} z(t)}{\mathrm{d} t}
$$

where $\Phi=N B l$ is the transformation factor and $N$ is the number of coil turns, $B$ is the magnetic flux density and $l$ is the effective length. The output voltage is:

$$
V_{m}(t)=V_{e m}-R_{c} i_{c}(t)-L_{c} \frac{\mathrm{d} i_{L}(t)}{\mathrm{d} t}
$$

where $R_{c}$ and $L_{c}$ are the resistance and inductance of the coil respectively and $i_{c}(t)$ is the current through the coil. The electromagnetic force is calculated as:

$$
F_{e m}=\Phi i_{c}(t)
$$

The resonant frequency of the tuned microgenerator $\left(f_{r}^{\prime}\right)$ is:

$$
f_{r}^{\prime}=f_{r} \sqrt{1+\frac{F_{t}}{F_{b}}}
$$

where $f_{r}$ is the un-tuned resonant frequency, $F_{t}$ is the tuning force between two magnets and $F_{b}$ is the buckling load of a cantilever. The numerical values of the microgenerator parameters are listed in Table I.

\section{TABLE I}

NUMERICAL VALUES OF MICROGENERATOR PARAMETERS

\begin{tabular}{|l|l|l||l|l|l|}
\hline Symbol & Value & Unit & Symbol & Value & Unit \\
\hline$m$ & $2.0 \mathrm{e}-3$ & $\mathrm{~kg}$ & $R_{c}$ & 850 & $\Omega$ \\
$c_{p}$ & $1.3 \mathrm{e}-3$ & $\mathrm{Nm}^{-1} \mathrm{~s}^{-1}$ & $L_{c}$ & 0.58 & $\mathrm{H}$ \\
$k_{s}$ & 172.87 & $\mathrm{Nm}^{-1}$ & $F_{b}$ & 0.5 & $\mathrm{~N}$ \\
$\Phi$ & 1.33 & $\mathrm{NA}^{-1}$ & & & \\
\hline
\end{tabular}

The resonant frequency of the microgenerator can be calculated from Table I and equation (15) can be calculated as $\omega_{0}=93.87 \frac{\mathrm{rad}}{\mathrm{sec}}$. Hence, from inequality (9), the step size limit for stable integration of the microgenerator equations with the 3rd order AB formula is $7.7 \mathrm{msec}$.

Equations (14), (17) and (18) can be rearranged and written in the state-space form as follows:

$$
\begin{aligned}
\frac{\mathrm{d}}{\mathrm{d} t}\left[\begin{array}{c}
\frac{\mathrm{d} z(t)}{\mathrm{d} t} \\
z(t) \\
i_{c}(t)
\end{array}\right] & =\left[\begin{array}{ccc}
\frac{-c_{p}}{m} & \frac{-k_{s}}{m} & \frac{-\Phi}{m} \\
1 & 0 & 0 \\
\frac{-\Phi}{L_{c}} & 0 & \frac{-R_{c}}{L_{c}}
\end{array}\right]\left[\begin{array}{c}
\frac{\mathrm{d} z(t)}{\mathrm{d} t} \\
z(t) \\
i_{c}(t)
\end{array}\right] \\
& +\left[\begin{array}{cc}
0 & 0 \\
0 & 0 \\
\frac{-1}{L_{c}} & 0
\end{array}\right]\left[\begin{array}{c}
V_{m} \\
I_{m}
\end{array}\right]+\left[\begin{array}{c}
0 \\
\frac{F_{a}-F_{t-z}}{m} \\
0
\end{array}\right]
\end{aligned}
$$

Model equations are implemented using the BuildM and BuildRhs functions according to the SystemC-A equation building rules [30]. 


\section{B. Power processing}

Two power processing circuits have been modeled and compared for power transfer efficiency, a 5-stage Dickson voltage multiplier (Fig. 4) [31] and a switching mode boost converter (Fig. 5) [32]. Both systems contain non-linear components, so it is necessary to linearize the model to produce linearized state-space equations. The linearized diode equation is produced by differentiating the Boltzmann equation: $I_{d}=$ $I_{s}\left(e^{V_{d} / V_{t}}-1\right)$ wrt the diode voltage $V_{d} . I_{d}$ is the diode current, $I_{s}$ - the saturation current and $V_{t}$ is the thermal voltage. The linearized form is: $I_{d}=G V_{d}+J$ where $G=\frac{d I_{d}\left(V_{d}\right)}{d V_{d}}$ and $J=I_{d}\left(V_{d}\right)$ are the values of the equivalent conductance and ideal current source at the current time point. As an additional measure to save CPU time, $G$ and $J$ can be precalculated as piece-wise linear functions of $V_{d}$ and stored in look-up tables for different values of $V_{d}$. The model is linear at each time point although $G$ and $J$ change. Due to the forward march-intime nature of explicit integration, the required Jacobian values can be retrieved from the look-up tables fast, without the need to evaluate the original, physical equations. To maintain high modeling accuracy the granularity of the piece-wise linear models can be arbitrarily fine since the size of the look-up tables does not affect the simulation speed.

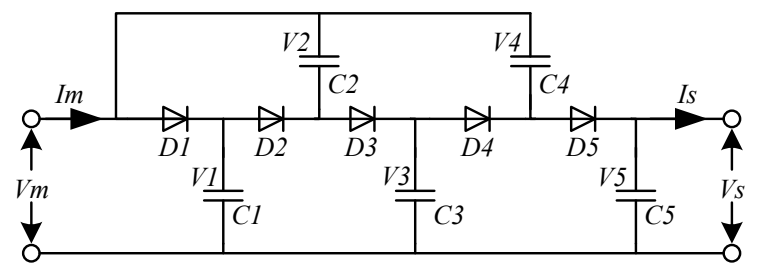

Fig. 4. 5-stage Dickson voltage multiplier as power processing circuit.

The state variables of the power processing circuit model are the voltages across each capacitor $\left(V_{1}\right.$ to $\left.V_{5}\right) . C_{i}, G_{i}$ and $J_{i}(i=1$ to 5$)$ are the corresponding capacitance, conductance and current values of the linearized model. $C_{1}$ to $C_{5}$ are $100 \mu \mathrm{F}$ and the diodes used in this section are all Schottky barrier diodes BAT85. The terminal variables are $V_{m}, I_{m}, V_{s}$ and $I_{s}$. The state equations are:

$$
\begin{aligned}
\frac{\mathrm{d}}{\mathrm{d} t}\left[\begin{array}{l}
V_{1} \\
V_{2} \\
V_{3} \\
V_{4} \\
V_{5}
\end{array}\right]= & {\left[\begin{array}{ccccc}
\frac{-G_{1}-G_{2}}{C_{1}} & \frac{-G_{2}}{C_{1}} & 0 & 0 & 0 \\
\frac{-G_{2}}{C_{2}} \frac{-G_{2}-G_{3}}{C_{2}} & \frac{-G_{3}}{C_{2}} & 0 & 0 \\
0 & \frac{-G_{3}}{C_{3}} & \frac{-G_{3}-G_{4}}{C_{3}} & \frac{-G_{4}}{C_{3}} & 0 \\
0 & 0 & \frac{-G_{4}}{C_{4}} & \frac{-G_{4}-G_{5}}{C_{4}} & \frac{-G_{5}}{C_{4}} \\
0 & 0 & 0 & \frac{-G_{5}}{C_{5}} & \frac{-G_{5}}{C_{5}}
\end{array}\right]\left[\begin{array}{l}
V_{1} \\
V_{2} \\
V_{3} \\
V_{4} \\
V_{5}
\end{array}\right] } \\
& +\left[\begin{array}{cccc}
\frac{G_{1}+G_{2}}{C_{1}} & 0 & 0 & 0 \\
\frac{G_{2}+G_{3}}{C_{2}} & 0 & 0 & 0 \\
\frac{G_{3}+G_{4}}{C_{3}} & 0 & 0 & 0 \\
\frac{G_{4}+G_{5}}{C_{4}} & 0 & 0 & 0 \\
\frac{G_{5}}{C_{5}} & 0 & 0 & \frac{-1}{C_{5}}
\end{array}\right]\left[\begin{array}{c}
V_{m} \\
I_{m} \\
V_{s} \\
I_{s}
\end{array}\right]+\left[\begin{array}{c}
\frac{J_{1}-J_{2}}{C_{1}} \\
\frac{J_{3}-J_{2}}{C_{2}} \\
\frac{J_{3}-J_{4}}{C_{3}} \\
\frac{J_{5}-J_{4}}{C_{4}} \\
\frac{J_{5}}{C_{5}}
\end{array}\right]
\end{aligned}
$$

As an alternative to the Dickson voltage multiplier, a switching-mode boost converter shown in Fig. 5 was investigated. The boost converter consists of a full-wave rectifier, an inductor, a diode, an output capacitor and a switch which is modeled as a variable resistor $R_{S W}$ :

$$
R_{S W}= \begin{cases}10.0^{9} \Omega & \text { when the switch is off } \\ 4 \Omega & \text { when the switch is on }\end{cases}
$$

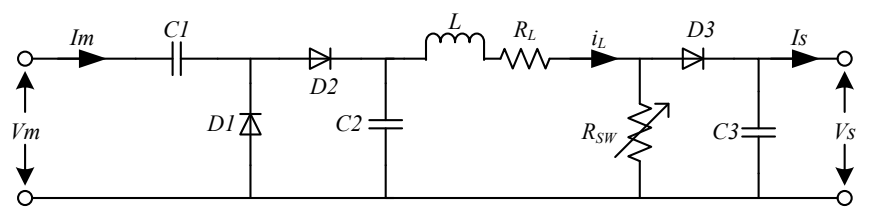

Fig. 5. Boost converter as power processing circuit.

The state variables here are the inductor current $i_{L}$ and voltages across each capacitor $\left(V_{1}\right.$ to $\left.V_{3}\right) . C_{i}, G_{i}$ and $J_{i}(i=1$ to 3$)$ are the capacitor, conductor and current values of the linearized model. The terminal variables are $V_{m}, I_{m}, V_{s}$ and $I_{s}$. The linearized state equations are:

$$
\begin{aligned}
& \frac{\mathrm{d}}{\mathrm{d} t}\left[\begin{array}{l}
V_{1} \\
V_{2} \\
V_{3} \\
i_{L}
\end{array}\right]=\left[\begin{array}{cccc}
0 & 0 & 0 & 0 \\
\frac{-G_{2}}{C_{2}} & \frac{-G_{2}}{C_{2}} & 0 & \frac{-1}{C_{2}} \\
0 & 0 & \frac{-G_{3}}{C_{3} X} & \frac{G_{3} R_{S W}}{C_{3} X} \\
0 & \frac{1}{L} & \frac{-G_{3} R_{S W}}{L X} & \frac{-R_{L} R-R_{S W}}{L X}
\end{array}\right]\left[\begin{array}{l}
V_{1} \\
V_{2} \\
V_{3} \\
i_{L}
\end{array}\right] \\
& +\left[\begin{array}{cccc}
0 & \frac{1}{C_{1}} & 0 & 0 \\
\frac{G_{2}}{C_{2}} & 0 & 0 & 0 \\
0 & 0 & 0 & \frac{-1}{C_{3}} \\
0 & 0 & 0 & 0
\end{array}\right]\left[\begin{array}{c}
V_{m} \\
I_{m} \\
V_{s} \\
I_{s}
\end{array}\right]+\left[\begin{array}{c}
\frac{J_{2}}{C_{2}} \\
\frac{J_{3}\left(X+G_{3} R_{S W}\right)}{C_{3} X} \\
\frac{J_{3} R_{S W}}{L X}
\end{array}\right]
\end{aligned}
$$

where $X=1+G_{3} R_{S W}$. The numerical values of the boost converter parameters are: $L=22 \mu \mathrm{H}, R_{L}=0.65 \Omega$ and $C_{i}=100 \mu \mathrm{F}$ $(i=1$ to 3$)$.

Direct application of explicit integration to solve equation (23) would lead to extremely small step sizes due to the low time constant of the inductor $L$ and the small ON resistance of the switch $R_{S W}$. Two techniques of avoiding small step sizes have been investigated and are discussed below. The first is the traditional state-space averaging technique developed by Middlebrook et. al [33]. In the second approach we propose to solve the inductor current analytically to avoid small step sizes.

a) State-space averaging: Since the proposed technique uses a state-space equation formulation, the classic state-space averaging method [33] is suitable to be adapted. The state equation of the inductor current is:

$$
\begin{aligned}
\frac{\mathrm{d} i_{L}(t)_{O N}}{\mathrm{~d} t} & =\frac{V_{2}-i_{L} R_{L}}{L} \\
\frac{\mathrm{d} i_{L}(t)_{O F F}}{\mathrm{~d} t} & =\frac{V_{2}-i_{L} R_{L}-V_{3}}{L}
\end{aligned}
$$

for the switch ON and OFF states correspondingly. With the PWM (pulse width modulation) duty cycle of $D$ and $D^{\prime}=$ $1-D$, the averaging state equation is:

$$
\frac{\mathrm{d} i_{L}(t)}{\mathrm{d} t}=D \frac{\mathrm{d} i_{L}(t)_{O N}}{\mathrm{~d} t}+D^{\prime} \frac{\mathrm{d} i_{L}(t)_{O F F}}{\mathrm{~d} t}
$$

Equation 26 replaces the last line in the matrix of equation (23) and the state-space averaging technique generates the "averaged" system behavior. There are several disadvantages of the state-space averaging technique. Because the switching behavior of the system is "averaged-out", it can only predict the system's long time behavior but not short time behavior such as the output ripple. Furthermore, the inductor size still limits the simulation time step and therefore the simulation speed. 
b) Symbolic solution of the inductor current: In this section, we consider the possibility of maintaining the high accuracy of the short time behavior by solving the inductor current symbolically to avoid excessive reductions of the simulation time step. The differential equation of the inductor current is:

$$
\frac{\mathrm{d} i_{L}(t)}{\mathrm{d} t}+\frac{i_{L}(t)}{\tau}=I_{0}
$$

where $I_{0}$ and $\tau$ are both constants. It is a first-order linear equation and its general solution is:

$$
i_{L}(t)=I_{0} \tau+C e^{-t / \tau}
$$

The initial condition is at $t=0, i_{L}(0)=i_{n}$, where $i_{n}$ is the present time-point value and hence $C=i_{n}-I_{0} \tau$. Therefore the solution of next time-point value $\left(i_{n+1}\right)$ is:

$$
i_{n+1}=i_{n}+\left(I_{0} \tau-i_{n}\right)\left(1-e^{-h / \tau}\right)
$$

where $h$ is the simulation time step. In the above boost converter case:

$$
\begin{gathered}
I_{0}=\frac{\left(V_{2}-V_{3}\right) X+J_{3} R_{S W}+V_{3}}{L X} \\
\tau=\frac{L X}{R_{L} X+R_{S W}}
\end{gathered}
$$

\section{Energy storage and load}

The supercapacitor equivalent circuit (Fig. 6) is comprised of a network of four RC circuits which model the charge redistribution process within the supercapacitor [34]. This model reflects the non-linear as well as multi-time-constant nature of the supercapacitor and reflects both fast and slow charge and discharge behavior.

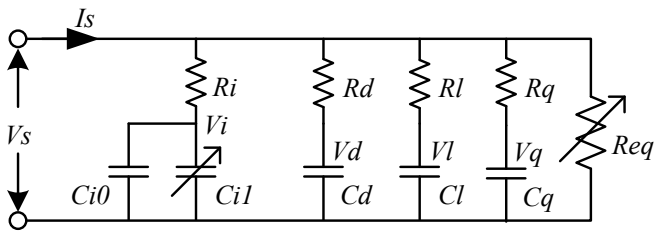

Fig. 6. Supercapacitor and equivalent load resistor model

The state variables of the supercapacitor model are the capacitor voltages $V_{i}, V_{d}, V_{l}$ and $V_{q}$. The terminal variables are $V_{s}$ and $I_{s}$. The state equations are:

$$
\frac{\mathrm{d}}{\mathrm{d} t}\left[\begin{array}{c}
V_{i} \\
V_{d} \\
V_{l} \\
V_{q}
\end{array}\right]=\left[\begin{array}{c}
\frac{-1}{R_{i}\left(C_{i 0}+C_{i 1}\right)} \\
0 \\
0 \\
0
\end{array}\right.
$$

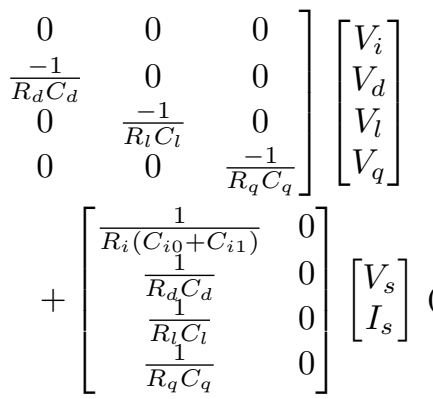

The supercapacitor used here is a GS206 0.55F from CAPXX (http://www.cap-xx.com/). The numerical values of the supercapacitor parameters are listed in Table II. $R_{e q}$ (Fig. 6) is the equivalent load resistor representing the power consumption of the linear actuator (Haydon 21000 Series Size 8 stepper motor, http://www.haydonkerk.com)and the microcontroller (PIC16F884). The $R_{e q}$ values for different operation modes were calculated from the components' datasheets:

$$
R_{e q}= \begin{cases}1.0 e 9 \Omega \text { when microcontroller is in sleep mode } \\ 33 \Omega & \text { when microcontroller wakes up } \\ 16.7 \Omega & \text { when actuator performs tuning }\end{cases}
$$

TABLE II

NUMERICAL VALUES OF SUPERCAPACITOR PARAMETERS

\begin{tabular}{|l|l|l||l|l|l|}
\hline Symbol & Value & Unit & Symbol & Value & Unit \\
\hline$C_{i 0}$ & 0.3962 & $\mathrm{~F}$ & $R_{i}$ & 0.742 & $\Omega$ \\
$C_{i 1}$ & 0.0956 & $\mathrm{~F}$ & & & \\
$C_{d}$ & 0.1392 & $\mathrm{~F}$ & $R_{d}$ & 305.4 & $\Omega$ \\
$C_{l}$ & 0.0654 & $\mathrm{~F}$ & $R_{l}$ & 15910 & $\Omega$ \\
$C_{q}$ & 0.0167 & $\mathrm{~F}$ & $R_{q}$ & 34375 & $\Omega$ \\
\hline
\end{tabular}

\section{Energy harvester digital control}

In order for a tunable energy harvester (Fig. 1) to work autonomously, both the actuator and the controller need to be powered by the stored energy. The time required for the harvester to generate enough energy to perform one frequency tuning process determines the harvester's duty cycle. The pseudo code of the tuning process is shown in Algorithm 1. Standard SystemC modules were used to model the digital control process and in the experimental verification the control algorithm was implemented in a PIC16F884 microcontroller. As can be seen in Algorithm 1, a watchdog timer wakes the microcontroller periodically and the microcontroller first detects if there is enough energy stored in the supercapacitor. If there is not enough energy, the microcontroller goes back to sleep and waits for the watchdog timer again. If there is enough energy, the microcontroller will then read the ambient vibration frequency from the accelerometer to see if it matches the microgenerator's resonant frequency. When a difference is detected between the vibration frequency and the resonant frequency, the microcontroller will start a tuning process by controlling the actuator to move the tuning magnet to the desired position (Fig. 3(a)). The accelerometer used in the experimental verification of this work was the MEMS inertial sensor type LIS3L06AL from STMicroelectronics.

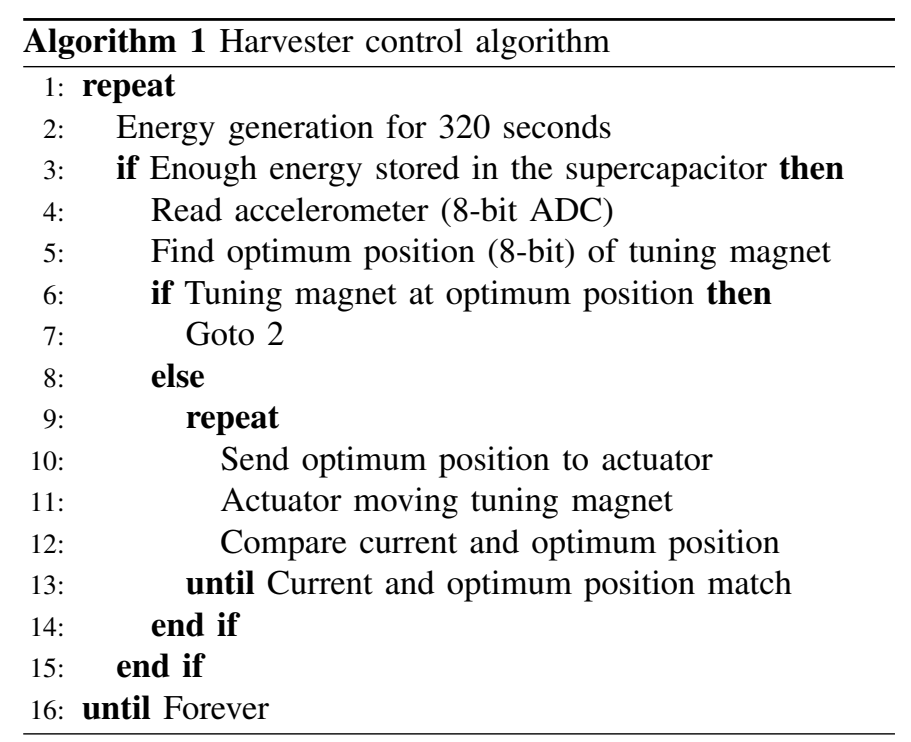




\section{E. Model of tunable energy harvester system}

When combining the three component blocks (microgenerator, power processing and supercapacitor) together, the terminal variables of each component block will be represented by state variables and eliminated. This enables the whole energy harvester model to be described by state equations and these state equations can be solved using explicit integration method, such as Adams-Bashforth. The complete set of linearized state equations of the system's analog part are generated by combining the equations of the three passive analog blocks. The combination is carried out by applying equation (13) to equations (20), (21) and (32). The complete equation set is:

$$
\mathrm{d}\left[\begin{array}{c}
\frac{\mathrm{d} z(t)}{\mathrm{d} t} \\
z(t) \\
i_{L}(t) \\
V_{1} \\
V_{2} \\
V_{3} \\
V_{4} \\
V_{5} \\
V_{i} \\
V_{d} \\
V_{l} \\
V_{q}
\end{array}\right]=\boldsymbol{J}\left[\begin{array}{c}
\frac{\mathrm{d} z(t)}{\mathrm{d} t} \\
z(t) \\
i_{L}(t) \\
V_{1} \\
V_{2} \\
V_{3} \\
V_{4} \\
V_{5} \\
V_{i} \\
V_{d} \\
V_{l} \\
V_{q}
\end{array}\right]+\left[\begin{array}{c}
\frac{F_{a}-F_{t z}}{m} \\
0 \\
\frac{J_{\Sigma}}{L_{c} G_{\Sigma}} \\
\frac{J_{1}-J_{2}}{C_{1}}-\frac{J_{\Sigma}}{\tau_{1} G_{\Sigma}} \\
\frac{J_{3}-J_{2}}{C_{2}}-\frac{J_{\Sigma}}{\tau_{2} G_{\Sigma}} \\
\frac{J_{3}-J_{3}}{C_{3}}-\frac{J_{\Sigma}}{\tau_{3} G_{\Sigma}} \\
\frac{J_{5}-J_{4}}{C_{4}}-\frac{J_{\Sigma}}{\tau_{4} G_{\Sigma}} \\
\frac{J_{5}}{C_{5}}-\frac{J_{\Sigma}}{\tau_{5} G_{\Sigma}} \\
0 \\
0 \\
0 \\
0
\end{array}\right]
$$

where $\boldsymbol{J}$ is the Jacobian of the combined equations (8), (20), (21) and (32).

\section{SimUlATION RESULTS AND EXPERIMENTAL VERIFICATION}

Two sets of simulations have been carried out. The first set of simulations aims to compare the two types of power processing circuits presented in Section III-B to see which one has a higher power transfer efficiency while working with the same microgenerator. The second set of simulations investigates two frequency tuning scenarios of the complete tunable energy harvesting system as outlined below. Simulation results are validated with experimental measurements.

\section{A. Comparison of power processing circuits}

Two SystemC-A models of the same microgenerator and different power processing circuits (one voltage multiplier and one boost converter) have been developed using the proposed linearized state-space technique and simulated. In both models, the frequency and amplitude of the input mechanical vibrations are fixed, i.e. no frequency tuning needed, and the supercapacitors are the same. The power transfer efficiency is defined as the output power divided by the input power. The input power is the product of input current and input voltage, both RMS values. The output power is calculated from the output DC voltage, i.e $P_{\text {out }}=V_{s} C \Delta V / \Delta t$, where $C$ is the supercapacitor value and $\Delta V$ is the voltage increase during time $\Delta t$. This is mainly due to practical constrains of the experimental setup (the input current to a supercapacitor is difficult to measure), and the simulation results are calculated from the same way for fair comparison. Fig. 7(a) shows the simulation results and Fig. 7(b) shows the experimental measurements.

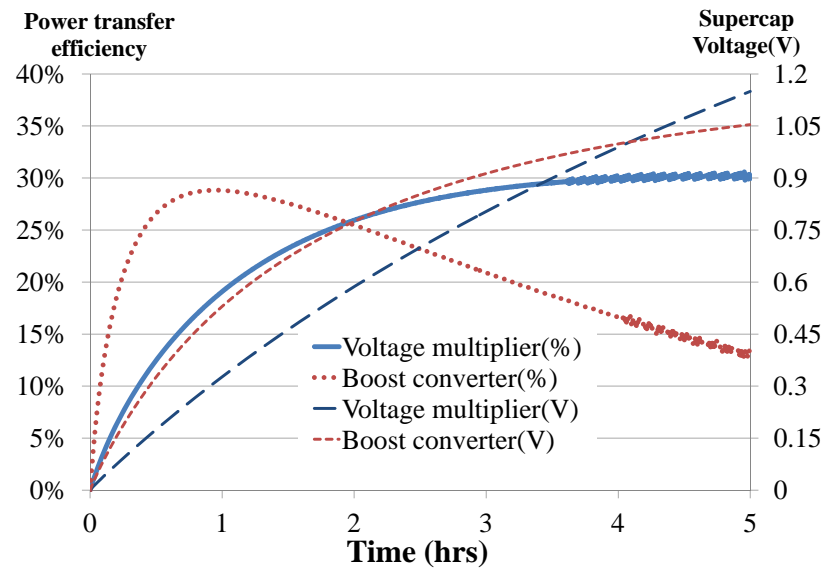

(a) Simulation results

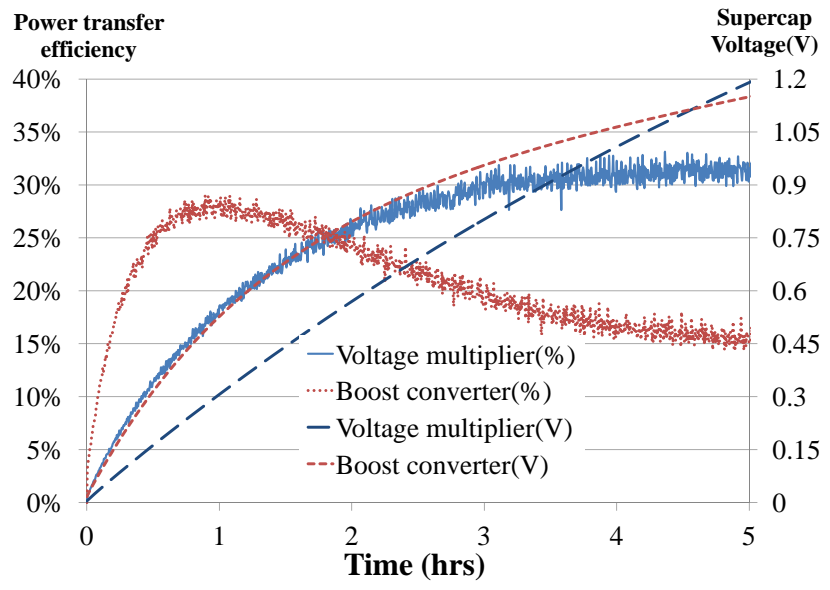

(b) Experimental measurements

Fig. 7. Simulations and experimental measurements of power transfer efficiency and supercapacitor voltage.

In both Fig. 7(a) and 7(b), the efficiency of the boost converter starts higher but drops quickly while the supercapacitor is being charged leading to a much lower overall efficiency than the voltage multiplier. The voltage multiplier charges the supercapacitor faster than the boost converter for voltages above $1 \mathrm{~V}$. The tuning actuator needs a supply voltage of $2.6 \mathrm{~V}$ and this is why the voltage multiplier is chosen as the power processing circuit in the next case study. We have observed that the main cause of the differences between the experimental measurements and simulation results is the inaccuracy of the supercapacitor model. By using the presented four-branch RC model of the supercapacitor, the maximum relative error is below $15 \%$. The higher the number of RC branches, the better the accuracy of the model, especially over long periods of charging and discharging. However, higher number of RC branches also means longer simulation CPU time. With a three-branch $\mathrm{RC}$ model, the maximum relative error is greater (about 30\%) and simulation CPU times are about $10 \%$ less compared with those shown in Tables III and IV. We have chosen four-branch model due to its good balance between accuracy and simulation CPU time. Simulation of the boost converter models described in Section III-B were also carried out and results are shown in Table III. The step size in standard SystemC-A was $0.1 \mu$ s and the resulting NewtonRaphson iteration count was 2.78 per step on average. The 
standard Newton-Raphson-based SystemC-A engine exhibits unstable behavior when the step-size is increased above $0.1 \mu \mathrm{s}$. With the symbolic solution for the inductor current outlined in Section III-B $b$ ), it was possible to increase the step-size up to $10 \mu$ s using the proposed state-space technique, except during the short switching intervals where the step-size was $0.1 \mu \mathrm{s}$. Note that the proposed linearized state-space technique is an explicit march-in-time process hence no iterations are involved at a time step.

TABLE III

CPU TIMES OF BOOST CONVERTER SIMULATIONS USING EXISTING AND PROPOSED SIMULATION TECHNIQUES IMPLEMENTED IN SYSTEMC-A

\begin{tabular}{|c|c|c|c|}
\hline $\begin{array}{c}\text { Circuit model } \\
\text { in Fig. 5 }\end{array}$ & $\begin{array}{c}\text { Standard } \\
\text { circuit }\end{array}$ & $\begin{array}{c}\text { State-space } \\
\text { averaging }\end{array}$ & $\begin{array}{c}\text { Inductor current } \\
\text { symbolic solution }\end{array}$ \\
\hline $\begin{array}{c}\text { Integration } \\
\text { method }\end{array}$ & $\begin{array}{c}\text { SystemC-A } \\
\text { standard engine }\end{array}$ & \multicolumn{2}{|c|}{$\begin{array}{c}\text { Proposed state-space } \\
\text { technique }\end{array}$} \\
\hline Step size & $0.1 \mu \mathrm{s}$ & $5 \mu \mathrm{s}$ & $0.1-10 \mu \mathrm{s}$ \\
\hline $\begin{array}{c}\text { Average no. } \\
\text { of iterations } \\
\text { per step }\end{array}$ & 2.78 & 1 & 1 \\
\hline CPU time & $\begin{array}{c}16 \text { days } \\
\text { (estimated) }\end{array}$ & 135 mins & $100 \mathrm{mins}$ \\
\hline
\end{tabular}

\section{B. Frequency tuning}

To investigate the efficiency of the simulation acceleration method presented in Section II, experimental verification has been carried out using a recently reported practical vibration energy harvester [29] (Fig. 1). We have chosen this harvester for our case study because it is a complete and the most recent autonomous tunable energy harvester presented in the literature [14]. The proposed linearized state-space technique and the mixed-technology model of the tunable energy harvester presented in the previous section have been implemented in SystemC-A. For comparison, simulations of the same tunable energy harvester using implicit, NewtonRaphson based integration in VHDL-AMS and SystemCA have also been carried out. Two scenarios have been tested. Scenario 1 is based on a narrow tuning range, varying the frequency by $1 \mathrm{~Hz}$, and Scenario 2 is based on a wide tuning range where the frequency varies by $14 \mathrm{~Hz}$ which is the maximum tuning range of the design [29]. Because the VHDL-AMS and SystemC-A models are based on the same equations and their simulation results are almost identical, only simulation waveforms generated by the SystemC-A model are compared with experimental measurements of the practical tunable energy harvester.

Fig. 8(a) shows the power output from the microgenerator during the $1 \mathrm{~Hz}$ tuning process. The waveform shows that when the ambient frequency shifts from 70 to $71 \mathrm{~Hz}$, as expected the output power drops down and goes up before and after tuning. The simulated RMS power is $118 \mu \mathrm{W}$ when the microgenerator is tuned at $70 \mathrm{~Hz}$ and $117 \mu \mathrm{W}$ when it is tuned at $71 \mathrm{~Hz}$. These values match well with the reported practical test value of $116 \mu \mathrm{W}$ as presented in [19]. Fig. 8(b) shows the comparison between simulated and experimental measured voltage waveforms across the supercapacitor of the energy harvester. As can be seen, the simulation waveform correlates well with the experimental measurement. In Scenario 2, we increase the frequency variation to $14 \mathrm{~Hz}$ which presents a more challenging simulation case due to the wider frequency range. Yet there is close correlation between simulation and experimental waveforms as can be seen in Fig. 9. This provides evidence that our technique is accurate even for energy harvester with a wide frequency tuning range. Whilst Fig. 8(b) and 9 show close agreements between the simulation and experimental results, there are some differences between the waveforms. This is because the HDL model of the energy harvester cannot incorporate every aspect of a practical system, such as leakage and parasitic loss.

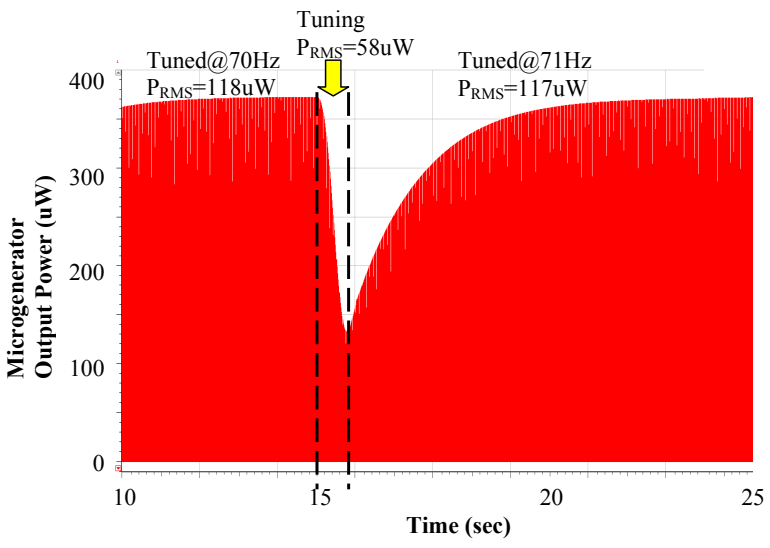

(a) Simulation result of output power from microgenerator

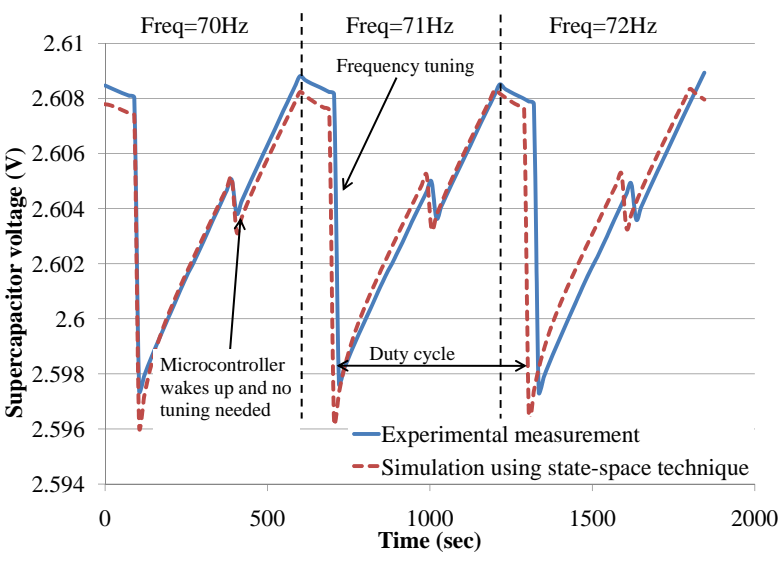

(b) Simulation and experimental results of supercapacitor voltage

Fig. 8. Scenario 1: Simulation and experimental results of $1 \mathrm{~Hz}$ tuning

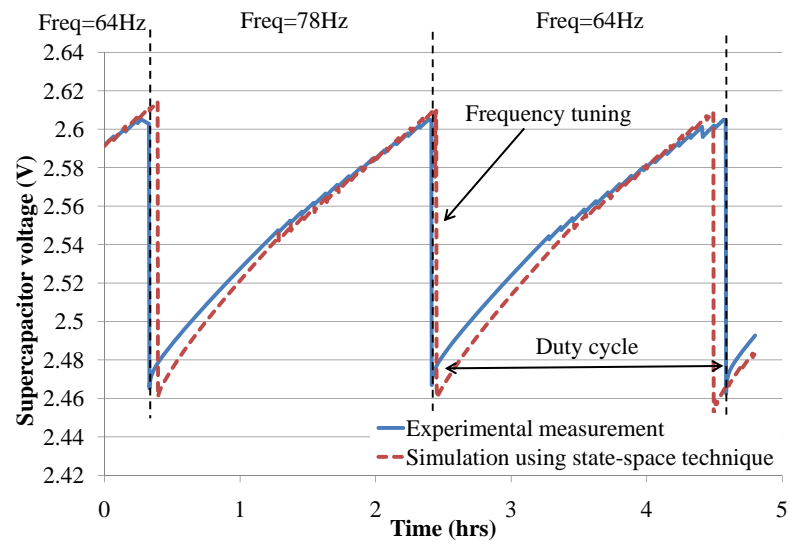

Fig. 9. Scenario 2: Simulation and experimental results of $14 \mathrm{~Hz}$ tuning 
Table IV shows CPU times of both the proposed state-space simulation and the Newton-Raphson simulation for the energy harvester in Scenario 1 and 2. It can be seen that the statespace technique can accelerate the simulation by two orders of magnitude compared to that of the existing simulator.

TABLE IV

CPU TIMES OF EXISTING AND PROPOSED SIMULATION TECHNIQUES

\begin{tabular}{|c|c|c|c|}
\hline & \multicolumn{2}{|c|}{ Existing technique } & Proposed technique \\
\hline $\begin{array}{c}\text { Integration } \\
\text { method }\end{array}$ & \multicolumn{2}{|c|}{$\begin{array}{c}\text { Implicit Newton- } \\
\text { Raphson based }\end{array}$} & $\begin{array}{c}\text { Explicit linearized } \\
\text { Adams-Bashforth }\end{array}$ \\
\hline HDL & VHDL-AMS & \multicolumn{2}{c|}{ SystemC-A } \\
\hline Scenario 1 & $2448 \mathrm{sec}$ & $3155 \mathrm{sec}$ & $23.3 \mathrm{sec}$ \\
\hline Scenario 2 & $\begin{array}{c}31250 \mathrm{sec} \\
(8.7 \text { hours })\end{array}$ & $\begin{array}{c}35390 \mathrm{sec} \\
(9.8 \text { hours })\end{array}$ & $247 \mathrm{sec}$ \\
\hline
\end{tabular}

\section{CONCLUSION}

This work is the first investigation into accelerating the simulation time of energy harvesting systems, an important type of systems employed in emerging applications. The main motivation for the research into fast simulation of energy harvesters is future development of an automated design approach by which the best topology and optimal parameters of energy harvester are obtained iteratively using multiple simulations. The proposed linearized state-space technique provides a vital step towards achieving this goal. Our experimental and analytical results presented here show that explicit integration of the linearized state-space equations can significantly decrease the simulation time of such systems whilst maintaining high simulation accuracy as demonstrated by excellent correlation between experimental and simulation results.

\section{ACKNOWLEDGMENT}

This work was supported by EPSRC, UK, grant number EP/G067740/1, (http://www.holistic.ecs.soton.ac.uk). The authors would like to thank Steve Beeby, Ivo Ayala-Garcia and Alex Weddell for their help in the experimental setup and measurements.

\section{REFERENCES}

[1] S. Ergen, A. Sangiovanni-Vincentelli, X. Sun, R. Tebano, S. Alalusi, G. Audisio, and M. Sabatini, "The tire as an intelligent sensor," Computer-Aided Design of Integrated Circuits and Systems, IEEE Transactions on, vol. 28, no. 7, pp. 941-955, 2009.

[2] J. A. Paradiso and T. Starner, "Energy scavenging for mobile and wireless electronics," Pervasive Computing, IEEE, vol. 4, no. 1 , pp. 18-27, 2005.

[3] K. Makihara, J. Onoda, and T. Miyakawa, "Low energy dissipation electric circuit for energy harvesting," Smart materials and structures, vol. 15, no. 5, pp. 1493-1498, 2006.

[4] D. Dondi, A. Bertacchini, D. Brunelli, L. Larcher, and L. Benini, "Modeling and optimization of a solar energy harvester system for self-powered wireless sensor networks," Industrial Electronics, IEEE Transactions on, vol. 55, no. 7, pp. 2759-2766, 2008.

[5] V. Raghunathan, A. Kansal, J. Hsu, J. Friedman, and M. Srivastava, "Design considerations for solar energy harvesting wireless embedded systems," in Information Processing in Sensor Networks, 2005. IPSN 2005. Fourth International Symposium on, 15 April 2005, pp. 457-462.

[6] E. Lawrence and G. Snyder, "A study of heat sink performance in air and soil for use in a thermoelectric energy harvesting device," in Thermoelectrics, 2002. Proceedings ICT '02. TwentyFirst International Conference on, 25-29 Aug. 2002, pp. 446449.
[7] M. Mi, M. Mickle, C. Capelli, and H. Swift, "RF energy harvesting with multiple antennas in the same space," Antennas and Propagation Magazine, IEEE, vol. 47, no. 5, pp. 100-106, 2005.

[8] S. Roundy, E. Leland, J. Baker, E. Carleton, E. Reilly, E. Lai, B. Otis, J. Rabaey, P. Wright, and V. Sundararajan, "Improving power output for vibration-based energy scavengers," Pervasive Computing, IEEE, vol. 4, no. 1, pp. 28-36, 2005.

[9] S. P. Beeby, M. J. Tudor, and N. M. White, "Energy harvesting vibration sources for microsystems applications," Measurement Science and Technology, vol. 17, no. 12, pp. R175-R195, December 2006.

[10] C.-C. Chen, C.-W. Kuo, and Y.-J. Yang, "Generating passive compact models for piezoelectric devices," Computer-Aided Design of Integrated Circuits and Systems, IEEE Transactions on, vol. 30, no. 3, pp. 464-467, 2011.

[11] G. Ottman, H. Hofmann, A. Bhatt, and G. Lesieutre, "Adaptive piezoelectric energy harvesting circuit for wireless remote power supply," Power Electronics, IEEE Transactions on, vol. 17, no. 5, pp. 669-676, 2002.

[12] H. Shao, C.-Y. Tsui, and W.-H. Ki, "A charge based computation system and control strategy for energy harvesting applications," in Circuits and Systems, 2006. ISCAS 2006. Proceedings. 2006 IEEE International Symposium on, 21-24 May 2006, pp. 29332936.

[13] P. Mitcheson, T. Green, E. Yeatman, and A. Holmes, "Architectures for vibration-driven micropower generators," Journal of Microelectromechanical Systems, vol. 13, no. 3, pp. 429-440, 2004.

[14] D. Zhu, J. Tudor, and S. Beeby, "Strategies for increasing the operating frequency range of vibration energy harvesters: a review," Measurement Science and Technology, vol. 21, no. 2, 2010.

[15] L. Wang, T. Kazmierski, B. Al-Hashimi, A. Weddell, G. Merrett, and I. Ayala-Garcia, "Accelerated simulation of tunable vibration energy harvesting systems using a linearised state-space technique," in Design, Test and Automation in Europe (DATE 2011), March 14-18, 2011, pp. 1267-1272.

[16] C. Williams, C. Shearwood, M. Harradine, P. Mellor, T. Birch, and R. Yates, "Development of an electromagnetic microgenerator," Circuits, Devices and Systems, IEE Proceedings, vol. 148, no. 6, pp. 337-342, 2001.

[17] R. Amirtharajah, J. Wenck, J. Collier, J. Siebert, and B. Zhou, "Circuits for energy harvesting sensor signal processing," in Design Automation Conference, 2006 43rd ACM/IEEE, 24-28 July 2006, pp. 639-644.

[18] L. Wang, T. Kazmierski, B. Al-Hashimi, S. Beeby, and D. Zhu, "An automated design flow for vibration-based energy harvester systems," in Design, Test and Automation in Europe (DATE 2009), April 20-24, 2009, pp. 1391-1396.

[19] D. Zhu, S. Roberts, J. Tudor, and S. Beeby, "Design and experimental characterization of a tunable vibration-based electromagnetic micro-generator," Sensors and Actuators A: Physical, vol. 158, no. 2, pp. 284-293, 2010.

[20] D. Hohlfeld, S. Matova, and R. van Schaijk, "System-level modeling and simulation of a frequency-tunable electrostatic energy harvester," in Thermal, Mechanical and Multi-Physics simulation and Experiments in Microelectronics and Microsystems, 2009. EuroSimE 2009. 10th International Conference on, 26-29 April 2009, pp. 1-7.

[21] M. Zhu, E. Worthington, and J. Njuguna, "Analyses of power output of piezoelectric energy-harvesting devices directly connected to a load resistor using a coupled piezoelectric-circuit finite element method," Ultrasonics, Ferroelectrics and Frequency Control, IEEE Transactions on, vol. 56, no. 7, pp. 13091317, 2009.

[22] L.-C. Blystad, E. Halvorsen, and S. Husa, "Piezoelectric mems energy harvesting systems driven by harmonic and random vibrations," Ultrasonics, Ferroelectrics and Frequency Control, 
IEEE Transactions on, vol. 57, no. 4, pp. 908-919, 2010.

[23] L.-D. Liao, P.-P. Chao, J.-T. Chen, W.-D. Chen, W.-H. Hsu, C.-W. Chiu, and C.-T. Lin, "A miniaturized electromagnetic generator with planar coils and its energy harvest circuit," Magnetics, IEEE Transactions on, vol. 45, no. 10, pp. 46214627, 2009.

[24] V. Acary, O. Bonnefon, and B. Brogliato, "Time-stepping numerical simulation of switched circuits within the nonsmooth dynamical systems approach," Computer-Aided Design of Integrated Circuits and Systems, IEEE Transactions on, vol. 29, no. 7, pp. 1042-1055, 2010.

[25] L. O. Chua and P. Y. Lin, Computer-Aided Analysis of Electronic Circuits: Algorithms and Computational Techniques. Prentice Hall Professional Technical Reference, 1975.

[26] D. Zwillinger, Handbook of differential equations, 2nd ed. 1250 Sixth Avenue, San Diego, CA 92101: Academic Press, Inc., 1989.

[27] R. Varga, Matrix Iterative Analysis. Prentice-Hall, 1962.

[28] R. J. LeVeque, Finite difference methods for ordinary and partial differential equations. Philadelphia: SIAM, 2007.

[29] I. A. Garcia, D. Zhu, J. Tudor, and S. Beeby, "Autonomous tunable energy harvester," in PowerMEMS 2009, 1-4 December 2009, pp. 49-52.

[30] H. Al-Junaid and T. Kazmierski, "Analogue and mixed-signal extension to systemc," IEE Proceedings on Circuits, Devices and Systems, vol. 152, no. 6, pp. 682-690, 2005.

[31] H. Yan, J. Macias Montero, A. Akhnoukh, L. de Vreede, and J. Burghartz, "An integration scheme for RF power harvesting," in Proc. STW Annual Workshop on Semiconductor Advances for Future Electronics and Sensors, November 17-18, 2005, pp. 64-66.

[32] E. Carlson, K. Strunz, and B. Otis, "A $20 \mathrm{mV}$ input boost converter with efficient digital control for thermoelectric energy harvesting," Solid-State Circuits, IEEE Journal of, vol. 45, no. 4, pp. 741-750, 2010.

[33] R. Middlebrook and S. Cuk, "A general unified approach to modelling switching-converter power stages," in Power Electronics Specialists Conference, June 8-10, 1976, pp. 18-34.

[34] L. Zubieta and R. Bonert, "Characterization of double-layer capacitors for power electronics applications," Industry Applications, IEEE Transactions on, vol. 36, pp. 199-205, 2000.

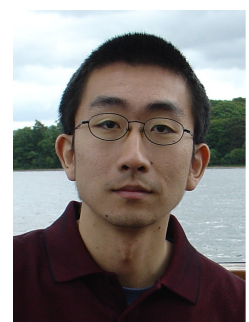

Leran Wang received his MSc degree from the University of Liverpool and $\mathrm{PhD}$ degree from University of Southampton, in 2004 and 2009 respectively. Before coming to UK, he received the BEng degree from Beijing University of Posts and Telecommunications in 2003. He is currently a research fellow at the School of Electronics and Computer Science, University of Southampton. His research interest focus on HDL based modelling and optimisation of mixed physical domain systems for energy harvesting, automotive and MEMS applications.

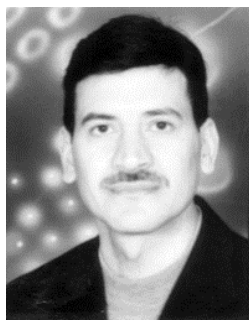

Bashir M. Al-Hashimi (M'99-SM'01-F'09) received the B.Sc. degree (1st-class classification) in Electrical and Electronics Engineering from the University of Bath, UK, in 1984 and the Ph.D. degree from York University, UK, in 1989. Following this he worked in the microelectronics design industry and in 1999, he joined the School of Electronics and Computer Science, University of Southampton, UK, where he is currently a Professor of Computer Engineering and Associate Dean (Research) of the Faculty of Physical and Applied Sciences. He has published over 240 papers in journals and refereed conference proceedings. Prof. Al-Hashimi is a Fellow of the IET and a Fellow of the British Computer Society. He is the Editor-in-Chief of the IEE Proceedings: Computers and Digital Techniques, and a member of the editorial board of the Journal of Electronic Testing: Theory and Applications (JETTA), and Journal of Low Power Electronics. He served as General Chair of the 11th IEEE European Test Symposium (UK 2006), Technical-Programme Chair of DATE 2009, and the General Chair of DATE 2011.

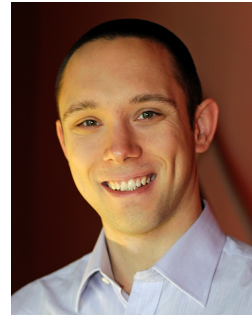

Geoff V. Merrett received the B.Eng. (1st class honours) degree in Electronic Engineering in 2004 and the Ph.D. degree in 2009 from the University of Southampton, where he is currently a lecturer in pervasive and embedded systems. He has authored a number of journal and conference publications in energy-aware wireless sensing and sensor networks, energy harvesting, modelling and simulation, and pervasive healthcare and co-edited a book on "Wireless Sensor Networks: Application-Centric Design (InTech, 2011)". Dr Merrett is a Co-Investigator on the EPSRC-funded projects "Next Generation Energy-Harvesting Electronics - A Holistic Approach" and "Energy Harvesting Network", and was awarded TSB funding for his interdisciplinary research on wearable devices for rehabilitation. 